\title{
Design and Development of the First Entirely Paper-Based Wireless Sensor Module
}

\author{
Rushi J. Vyas*, Amin Rida, Li Yang, Manos M. Tentzeris \\ School of Electrical and Computer Engineering, Georgia Institute of Technology, \\ Atlanta, GA, USA \\ E-mail: Rushi.Vyas@gatech.edu
}

\section{Introduction}

As the demand for low-cost, flexible and power-efficient broadband wireless electronics increase, the materials and integration techniques become more and more critical and face more challenges, especially with the ever increasing interest for "cognitive intelligence" and "ubiquitous wireless networks". This paper introduces the FIRST EVER wireless sensor module built and integrated COMPLETELY on paper. Specifically, a paper-based microcontroller enabled wireless sensor prototype has been developed for the first time for the UHF frequency band (centered at 904.4 MHz).

Paper is considered one of the best organic-substrate candidates for UHF and microwave applications for many reasons. It features the industrial ability to be processed in a reelto-reel fashion. Paper can also make utilization of direct write methodologies, such as inkjet-printing, to be used in replacement of relatively expensive wet-etching techniques. Such a fast process can be used to print electronics on the surface of paper substrate or even embedded in a multilayer fashion. Last, but not least, paper is one of the most environmentally-friendly materials and the proposed module could potentially set the foundation for the first generation of truly "green" RF electronics and modules.

\section{System Level Design}

This paper presents the first attempt ever made at integrating wireless transmitters with an organic photo-paper based substrate. The system comprises of a microcontroller enabled wireless sensor module on the paper with a topology shown in figure 1 . In our first prototype, the primary controller of the entire operation was an integrated 8-bit integrated microcontroller unit that was programmed to sample an external analog temperature sensor, process the analog information into its final miller bit-encoded digital form used in RFID communication [2], and transmit the digital version of the sensor information sequentially by amplitude modulating the power amplifier in the integrated transmitter module. The transmission frequency of $904.4 \mathrm{MHz}$ was generated by using a crystal oscillator that was tied to the input of the PLL unit of the transmitter. The data transmission was to be carried out at the unlicensed UHF frequencies around $900 \mathrm{MHz}$. The entire wireless system including the antenna was printed on a 2-D paper module and operated remotely using a $3 \mathrm{~V} \mathrm{Li-ion} \mathrm{cell.} \mathrm{The} \mathrm{design} \mathrm{steps} \mathrm{have} \mathrm{been} \mathrm{outlined} \mathrm{below.}$

\section{Amplifier Characterization}

In order to maximize the range of the wireless sensor module, and minimize components used for antenna matching, the antenna was designed to have an input impedance equal to the optimum impedance looking out of the power amplifier in the integrated transmitter 
module. At this optimum output load impedance the power amplifier gave out maximum output power, which maximized range. The optimum impedance looking out of the power amplifier were determined using load-pull techniques at frequencies of $860,904.4$ and $925 \mathrm{MHz}$ all within the unlicensed ISM band at UHF frequencies for a fixed bias point. The RF tuner used for the load-pull analysis was the Maury model 1643-N. The results and the circuit setup used for the load pull analysis are shown in table 1 and figure 2 respectively. The loss in the measurement setup i.e. the RF tuner and the serial cables were measured separately at each of the three measurement frequencies and added to the power output measured with the power meter to get the optimum output power shown in table 1. The effects of the SMA connector, CPW feed, the series capacitor and bias circuitry in between the amplifier in the transmitter IC and the SMA output shown in figure 2 were de-embedded out using Agilent's Advanced Design System software to get the optimum load impedance at the point looking out of the amplifier in the IC. This deembedded optimum output impedance of the power amplifier $\left(\mathrm{Z}_{\mathrm{L} \text {-opt }}\right)$ is what is shown in table 1.

Table 1. Load-pull measurements for Power Amplifier characterization.

\begin{tabular}{|l|l|l|l|}
\hline $\begin{array}{l}\text { Freq. } \\
(\mathrm{MHz})\end{array}$ & $\begin{array}{l}\mathrm{Z}_{\mathrm{L}-\mathrm{opt}} \\
\text { at IC }(\mathrm{ohms})\end{array}$ & \multicolumn{2}{|l|}{ Output Power- } \\
Measured $(\mathrm{dBm})$ & $\begin{array}{l}\text { Output Power- Manufacturer. } \\
(\mathrm{dBm})\end{array}$ \\
\hline 860 & $117.5+\mathrm{j} 137.14$ & 6.1 & 7.5 \\
\hline 904.4 & $60.1-\mathrm{j} 73.51$ & 7.43 & 7.5 \\
\hline 925 & $35.9-\mathrm{j} 15.87$ & 7.72 & 7.5 \\
\hline
\end{tabular}

\section{Antenna Design}

The antenna was designed to resonate at a center frequency of $904.4 \mathrm{MHz}$, where it would have an input impedance of $37+\mathrm{j} 22 \mathrm{ohms}$ and would be matched to the optimum output impedance of the power amplifier in the transmitter module. This aggregate impedance was determined after accounting for the shunt impedance introduced by the bias circuit and the series impedance introduced by the series coupling capacitor between the amplifier $\left(\mathrm{Z}_{\mathrm{L}-\mathrm{OPT}}=60.1-\mathrm{j} 73.51 \mathrm{ohms}\right.$ as shown in table 1$)$ and the antenna terminals at $904.4 \mathrm{MHz}$. The antenna used for this design was designed and optimized using Ansoft's HFSS 3-D EM solver. The antenna shape chosen was a U-shaped tapered dipole [1] to reduce the overall size (all other electronics can be printed/positioned within the area of the antenna in the interior of the "U-shape") while at the same time achieving a wide bandwidth near the design center frequency. The U-shape tapered structure shown in figure 6 is the antenna with overall structure dimensions: $9.5 \times 5 \mathrm{~cm}$. The Return Loss or S11 measurements for the center frequency for the antenna terminals was recorded to be $-15.05 \mathrm{~dB}$ for the simulated structure using the full wave EM simulator HFSS (figure 4) and $-12.45 \mathrm{~dB}$ measured using the ZVA-8 VNA. The radiation pattern was also measured using Satimo's Stargate 64 Antenna Chamber measurement system and by using the NIST Calibrated SH8000 Horn Antenna as a calibration kit for the measured radiation pattern at $904 \mathrm{MHz}$, and is shown in figure 3.

\section{Fabrication Assembly}

After the antenna was designed and characterized and the microcontroller was programmed the overall system performance was verified on the prototype circuit shown in figure 2; then, the complete design was reproduced on paper using inkjet printing technology. The antenna and the circuit layout were printed and cured on paper using 
silver ink and the complete wireless sensor system comprising the IC (MCU+ transmitter), its discrete passive components, the temperature sensor, and a Li-ion cell for "stand-alone" (autonomous) operation were assembled on it.

The inkjet-printing was done in a horizontal bar by bar printing. Two different cartridge settings were used, the slow but highly accurate setting for the quality of the small traces around the IC chip, and the low resolution but fast setting for the bulk printing of the UHF antenna [3]. Given the slow curing time of silver and silver epoxy and the accurate placement required of the IC ( $\sim 150$ microns pad spacing), the assembly was done using a 2 part process as shown in figure 5. The first step involved holding the components in place on the printed silver pads with the use of a very small amount of adhesive between the component terminals and the printed silver pads. Once in place, conductive silver epoxy was them applied on the terminals of the components to establish a conductive path between the terminals and printed silver pads. The complete assembly of all components also had to be done using a multi-step process. This was because the conductivity of the silver epoxy had a direct co-relation to its curing time and temperature, which contradicted with the limited temperature tolerance of the components used in the wireless transmitter. The final assembled entirely paper-based wireless sensor prototype can be seen in figure 6 .

\section{Results}

The sensor prototype was tested to study the effects of the packaging material and the series silver ink/silver epoxy interface on the sensor reading. The results can be seen in figure 7 featuring a very good agreement with the manufacturer's specs. The prototype was also tested for wireless transmission using a XR-400 RFID reader antenna interfaced to a Tektronix RSA 3408A Real Time Spectrum Analyzer (RTSA). The measured power spectrum can be seen in figure 8 and was observed to be $-69 \mathrm{dBm}$ at a low distance of 1 foot at a center frequency of $904.4 \mathrm{MHz}$.

\section{Conclusion}

In this paper, the feasibility of inkjet printing process for the development of a "Systemon-Paper" RF system was demonstrated for the first time. The process of designing a wireless transmitting sensor module entirely on paper was outlined and its performance was measured. The measured sensor results show good agreement with the manufacturer's specs. The relatively higher heat insulation of paper and silver ink to temperature changes compared to other conductors and packaging materials makes it an ideal substrate for housing sensors, as well as numerous "green" RF modules.

\section{References}

[1] A. Rida, L. Yang, and M. M. Tentzeris, "Design and characterization of novel paperbased inkjet-printed UHF antennas for RFID and sensing applications

" Procs. of the 2007 IEEE-APS Symposium, pp2749-2752, Honolulu, HI, July 2007

[2] "UHF Gen-2 System Overview". Texas Instruments, Sept 2005.

[3] L. Yang, A. Rida, R. Vyas, M. M. Tentzeris, "RFID Tag and RF Structures on a Paper Substrate Using Inkjet-Printing Technology," Microwave Theory and Techniques, IEEE Transactions on Volume 55, Issue 12, Part 2, Dec. 2007 Page(s):2894 - 2901 


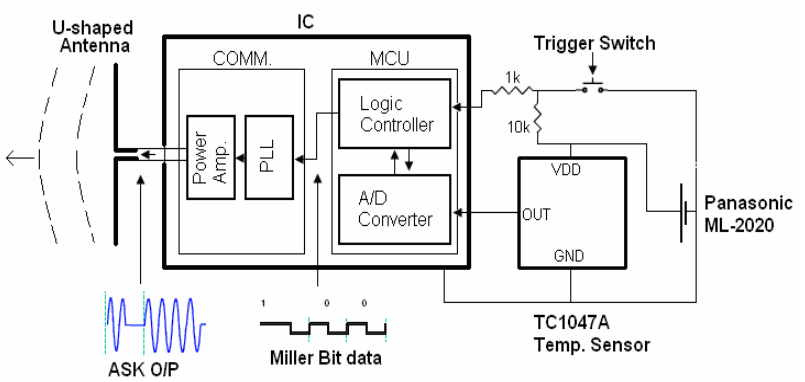

Fig 1.Wireless Sensor Module System.

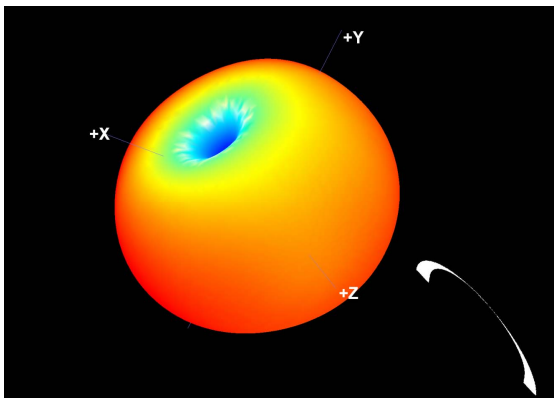

Fig 3. Measured antenna radiation pattern.

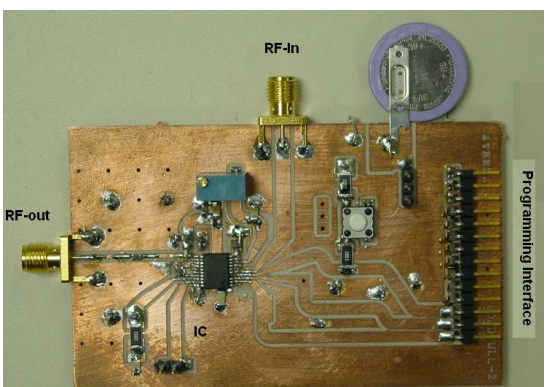

Fig 2. Prototyping board for load-pull analysis \& programming.

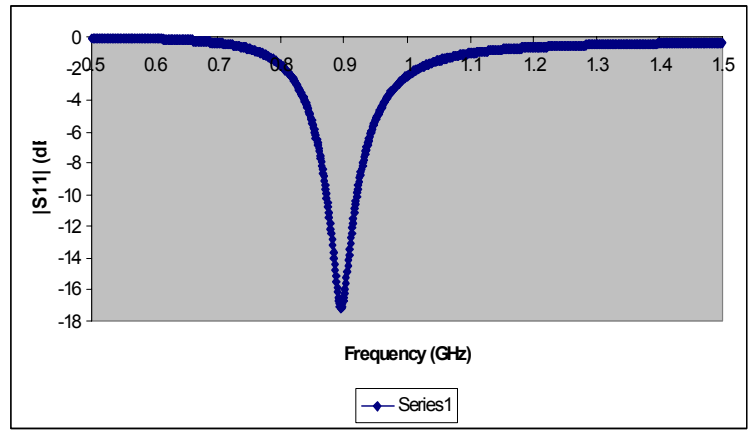

Fig 4. Simulated Antenna return Loss.

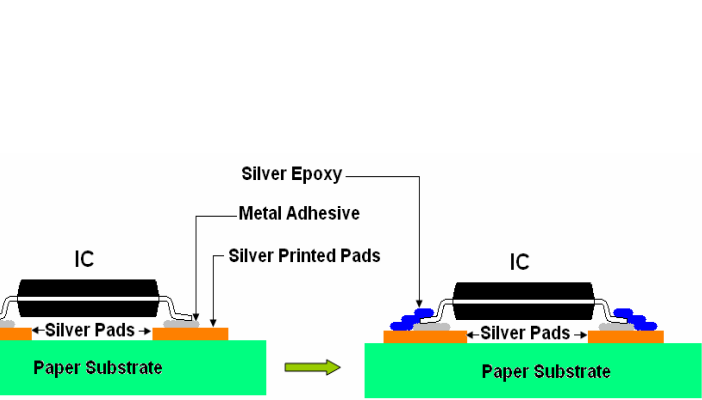

Fig 5. Two step assembly process.

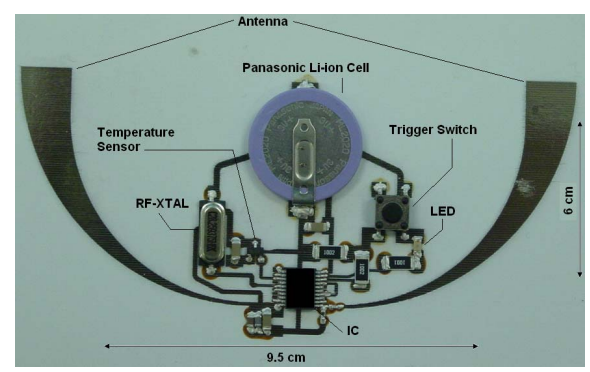

Fig 6. Final prototype Assembly.
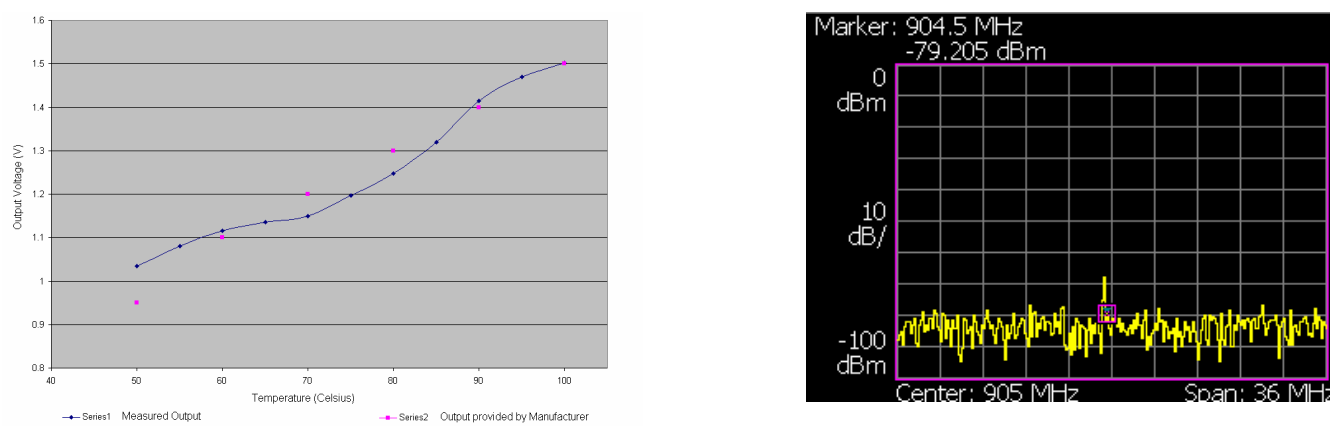

Fig 7. Sensor Performance. Measured vs. Manufacturer's specifications.

Fig 8. RTSA measured power level emitted by wireless sensor module. 